\title{
A new design for cubic spatially processed wideband arrays
}

\author{
Mohammad Ghavami ${ }^{1}$ iD
}

Received: 13 September 2018 / Revised: 24 December 2018 / Accepted: 18 February 2019 /

Published online: 26 February 2019

(c) The Author(s) 2019

\begin{abstract}
This paper presents a simple and novel formulation for the design and analysis of a generic cubic array with a single real coefficient for each antenna element, to steer the developed beam toward a certain desired angle in both azimuth (totally $360^{\circ}$ ) and elevation (totally $180^{\circ}$ ) directions. This configuration demonstrates frequency invariance of the directional patterns of the array within a relatively large fractional bandwidth which makes this array a potential candidate for wideband and ultra wideband applications. The required real coefficients are calculated based on matrix manipulation methods. However, the dimension of the matrices and vectors used in the computations are much less than the total number of antenna elements. Computer simulation shows the performance of the array with respect to the resolution of the main beam and frequency independence of the patterns and also in terms of the complexity and frequency range. Moreover, we will present a comparison between the proposed algorithm and the Fourier transform based methods and the adaptive least mean square algorithm.
\end{abstract}

Keywords Array signal processing $\cdot$ Wideband arrays $\cdot$ Spatial processing $\cdot$ Cubic arrays

\section{Introduction}

Wideband beam formation has attracted interest due to its potential applications in different areas such as radar, medical imaging and telecommunications (Liu 2010; Liang and Hum 2011). In conventional wideband beamforming methods that use both time and space domains, the wideband characteristics of the array is realized by applying the received signal of each antenna element to a pre-designed set of adjustable filters (Hawes and Liu 2014) or pure time delays (Allen and Ghavami 2005). In some other approaches which do not employ time-domain filters or delay elements, only a single real-values coefficient is used for each antenna element (Ghavami 2002).

Distributed beamforming methods using three dimensional volumetric arrays present higher flexibility in terms of throughput and bandwidth. In the analysis presented in Overturf et al. (2017) different array topologies including cubic configuration have been considered. As expected, this work concludes that larger volumes of omnidirectional elements produce narrower radiation patterns. As an application of cubically located sensor arrays, the detection

$\bowtie$ Mohammad Ghavami

ghavamim@1sbu.ac.uk

1 School of Engineering, London South Bank University, 103 Borough Road, London SE1 0AA, UK 
of a far field particle source has been studied in Srikanth et al. (2017). In another investigation, a cubic scanning array of antenna elements have been modelled and simulated. The behaviour of the resultant array is compared with the multiple planar array structure (Zhao and Peng 2009).

Cubic antenna arrays for wideband beamforming in both full azimuth and elevation directions using purely spatial processing has rarely been discussed in literature. In the method described in Liu (2010) and Liu and Weiss (2008), Fourier transform techniques have been used to calculate the weights connected to each element. In another approach, steerable cubic antenna arrays are designed by optimization of the amplitude and phase excitations across the elements (Garza et al. 2016). The novelty of this method is the application of an evolutionary optimization algorithm to a design problem.

In this paper a new algorithm is proposed to calculate the real multipliers of a linear cubic array. The main advantage of this method is the simplicity of the computation which does not involve two dimensional Fourier transform normally used in wideband array calculations. Moreover, it covers $360^{\circ}$ of azimuth and $180^{\circ}$ of elevation angles with a single structure. We also notice that the proposed method is superior in terms of overall size and frequency independence compared to the distributed technique presented in Overturf et al. (2017) which is using both temporal and spatial signal processing methodology. To compare our method with Srikanth et al. (2017), we observe that in this reference the frequency dependence of the beam patterns is not taken into account during the design process. This lack of discussion on the frequency dependency of the phased array is also a drawback of the method presented in Zhao and Peng (2009).

The rest of this paper is organized as follows. In Sect. 2 we will introduce the cubic array formulation. Section 3 explains the design of coefficients. Section 4 presents some computer simulation results including a comparison between the proposed algorithm and two dimensional Fourier transform method of Liu and Weiss (2008) and adaptive least mean square (LMS) algorithm (Widrow and Stearns 1985). Finally, Sect. 5 concludes the paper.

\section{Cubic array formulation}

Figure 1 shows the placement of the receiving antenna elements on the cubic volume. There are a total of $N_{1} \times N_{2} \times N_{3}$ elements located on three perpendicular $(x, y, z)$ directions. The distances between the elements along these directions are $d_{1}, d_{2}$ and $d_{3}$, respectively. The incoming wave arrives with the azimuth and elevation angles of $0^{\circ}<\theta<360^{\circ}$ and $-90^{\circ}<\phi<90^{\circ}$, respectively. The received signal at the array reaches the sample element $\left(n_{1}, n_{2}, n_{3}\right)$, for $0 \leq n_{1} \leq N_{1}-1,0 \leq n_{2} \leq N_{2}-1$ and $0 \leq n_{3} \leq N_{3}-1$, with a time delay that can be calculated as:

$$
\tau\left(n_{1}, n_{2}, n_{3}\right)=\frac{1}{c}\left[d_{1} n_{1} \cos \theta \sin \phi+d_{2} n_{2} \sin \theta \sin \phi+d_{3} n_{3} \cos \phi\right]
$$

Equation (1) has been calculated with respect to the reference element $(0,0,0)$ and $c$ is the speed of propagation. The frequency dependent phase angle at the location of the element $\left(n_{1}, n_{2}, n_{3}\right)$ will be calculated as follows:

$$
\phi\left(n_{1}, n_{2}, n_{3}\right)=2 \pi f \tau\left(n_{1}, n_{2}, n_{3}\right)
$$



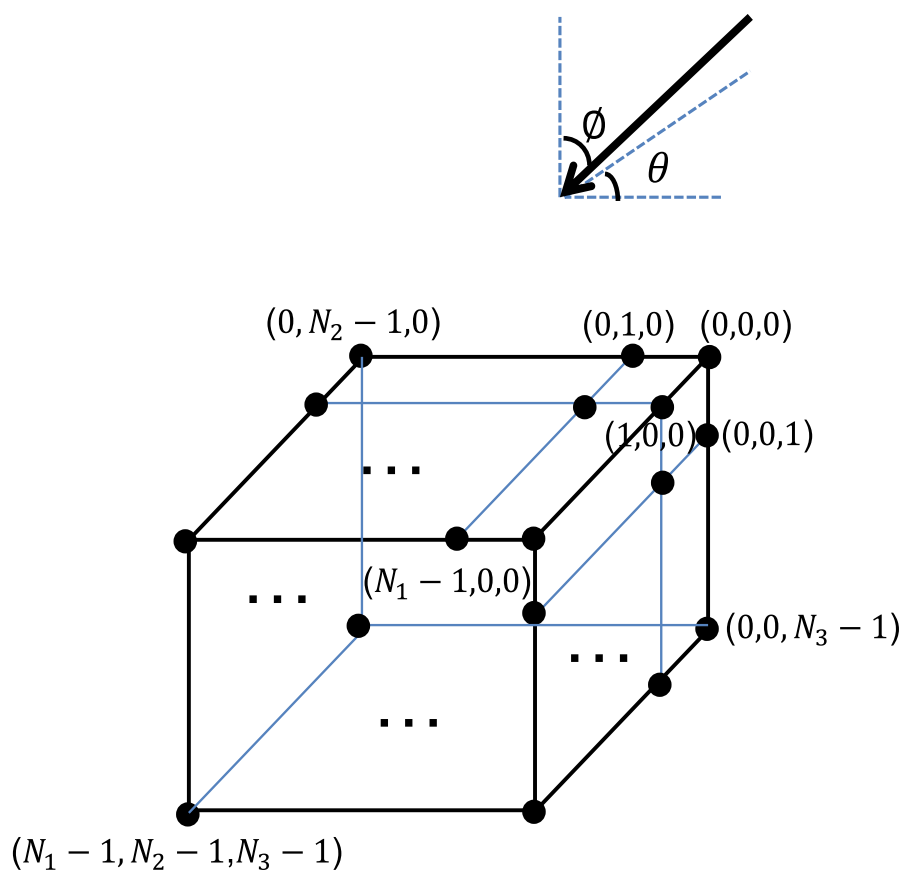

Fig. 1 Location of the antenna elements on the cubic volume with the incoming wave arriving at the azimuth and elevation angles of $\theta$ and $\phi$, respectively

The signal received at each element is multiplied by $C_{n_{1} n_{2} n_{3}}$ and the overall transfer function in terms of angle and frequency can be written as:

$$
H(f, \theta, \phi)=\sum_{n_{1}=0}^{N_{1}-1} \sum_{n_{2}=0}^{N_{2}-1} \sum_{n_{3}=0}^{N_{3}-1} C_{n_{1} n_{2} n_{3}} e^{j \frac{2 \pi f}{c}\left(d_{1} n_{1} \cos \theta \sin \phi+d_{2} n_{2} \sin \theta \sin \phi+d_{3} n_{3} \cos \phi\right)}
$$

For simplicity of analysis, three different frequencies are defined as:

$$
f_{1}=\frac{f d_{1}}{c} \cos \theta \sin \phi, \quad f_{2}=\frac{f d_{2}}{c} \sin \theta \sin \phi, \quad f_{3}=\frac{f d_{3}}{c} \cos \phi
$$

which simplifies (3) to the following form:

$$
H\left(f_{1}, f_{2}, f_{3}\right)=\sum_{n_{1}=0}^{N_{1}-1} \sum_{n_{2}=0}^{N_{2}-1} \sum_{n_{3}=0}^{N_{3}-1} C_{n_{1} n_{2} n_{3}} e^{j 2 \pi\left(n_{1} f_{1}+n_{2} f_{2}+n_{3} f_{3}\right)}
$$

The three dimensional frequency volume $\left(f_{1}, f_{2}, f_{3}\right)$ is limited for $f_{1}, f_{2}$ and $f_{3}$ to $(-0.5,0.5)$, because

$$
\begin{aligned}
& \left|f_{1}\right|=\left|\frac{f d_{1}}{c} \cos \theta \sin \phi\right| \leq \frac{f d_{1}}{c} \leq \frac{f}{c} \frac{\lambda_{\text {min }}}{2}=\frac{f}{c} \frac{c}{2 f_{h}} \leq 0.5 \\
& \left|f_{2}\right|=\left|\frac{f d_{2}}{c} \sin \theta \sin \phi\right| \leq \frac{f d_{2}}{c} \leq \frac{f}{c} \frac{\lambda_{\text {min }}}{2}=\frac{f}{c} \frac{c}{2 f_{h}} \leq 0.5 \\
& \left|f_{3}\right|=\left|\frac{f d_{3}}{c} \cos \phi\right| \leq \frac{f d_{3}}{c} \leq \frac{f}{c} \frac{\lambda_{\text {min }}}{2}=\frac{f}{c} \frac{c}{2 f_{h}} \leq 0.5
\end{aligned}
$$


where $\lambda_{\min }$ is the wavelength corresponding to the highest frequency $f_{h}$, and as a sensible choice we assume that the inter-element spacing is limited to the half of the lowest wavelength of the spectrum.

\section{Calculations of coefficients}

For the calculation of the real coefficients $C_{n_{1} n_{2} n_{3}}$, we start with considering $L \geq 2$ focal points symmetrically located on the three dimensional space generated by $\left(f_{1}, f_{2}, f_{3}\right)$. These points do not include the origin. Then, two vectors, both with the length of $L$, are defined as follows:

$$
\begin{aligned}
\mathbf{b} & =\left[b_{1}, b_{2}, \ldots, b_{L}\right]^{T} \\
\mathbf{H}_{\mathbf{0}} & =\left[H\left(f_{1_{0_{1}}}, f_{2_{0_{1}}}, f_{3_{0_{1}}}\right), \ldots, H\left(f_{1_{0_{L}}}, f_{2_{0_{L}}}, f_{3_{0_{L}}}\right)\right]^{T}
\end{aligned}
$$

where the superscript ${ }^{T}$ stands for transpose. The elements of the vector $\mathbf{H}_{\mathbf{0}}$ have the same values for any two pairs $\left(f_{1_{0_{l}}}, f_{2_{0_{l}}}, f_{3_{0_{l}}}\right), l=1,2, \ldots, L$ which are symmetrical with respect to the origin of the $\left(f_{1}, f_{2}, f_{3}\right)$ space. The vector $\mathbf{b}$ is an auxiliary vector and will be computed in the design process. Now, assume that $H\left(f_{1}, f_{2}, f_{3}\right)$ is expressed by the multiplication of three basic polynomials and then the summation of the weighted result by $b_{l}$ as follows:

$$
H\left(f_{1}, f_{2}, f_{3}\right)=\sum_{l=1}^{L} b_{l} \sum_{n_{1}=0}^{N_{1}-1} e^{j 2 \pi n_{1}\left(f_{1}-f_{1_{0}}\right)} \sum_{n_{2}=0}^{N_{2}-1} e^{j 2 \pi n_{2}\left(f_{2}-f_{20_{l}}\right)} \sum_{n_{3}=0}^{N_{3}-1} e^{j 2 \pi n_{3}\left(f_{3}-f_{30_{l}}\right)}
$$

We notice that by using this form of $H\left(f_{1}, f_{2}, f_{3}\right)$, we have reduced the problem of direct computation of $N_{1} N_{2} N_{3}$ coefficients $C_{n_{1} n_{2} n_{3}}$ from a complicated system of $N_{1} N_{2} N_{3}$ equations to a new problem of solving only $L$ equations, because normally we select $L<<N_{1} N_{2} N_{3}$. Anyhow, our final task will be finding $C_{n_{1} n_{2} n_{3}}$ from $b_{l}$. We can obtain the relationship between $b_{l}$ and $C_{n_{1} n_{2} n_{3}}$ be rearranging (11) as follows:

$$
\begin{aligned}
& H\left(f_{1}, f_{2}, f_{3}\right)= \sum_{n_{1}=0}^{N_{1}-1} \sum_{n_{2}=0}^{N_{2}-1} \sum_{n_{3}=0}^{N_{3}-1}\left\{\sum_{l=1}^{L} b_{l} e^{-j 2 \pi n_{1} f_{1_{0}}} e^{-j 2 \pi n_{2} f_{2_{0_{l}}}} e^{-j 2 \pi n_{3} f_{3_{l}} l}\right\} . \\
& e^{j 2 \pi n_{1} f_{1}} e^{j 2 \pi n_{2} f_{2}} e^{j 2 \pi n_{3} f_{3}}
\end{aligned}
$$

Comparing with (5) yields

$$
C_{n_{1} n_{2} n_{3}}=\sum_{l=1}^{L} b_{l} e^{-j 2 \pi n_{1} f_{1_{0}}} e^{-j 2 \pi n_{2} f_{2_{0}}} e^{-j 2 \pi n_{3} f_{3_{0}}}
$$

After calculation of $\mathbf{b},(13)$ provides a formula to determine all coefficients $C_{n_{1} n_{2} n_{3}}$. The computation of $\mathbf{b}$ is done using (11). For this purpose, we define an $L \times L$ matrix $\mathbf{A}$ with the elements $a_{k l}, 1 \leq k, l \leq L$ as follows:

$$
a_{k l}=\sum_{n_{1}=0}^{N_{1}-1} e^{j 2 \pi n_{1}\left(f_{1_{0}}-f_{1_{0}}\right)} \sum_{n_{2}=0}^{N_{2}-1} e^{j 2 \pi n_{2}\left(f_{2_{0}}-f_{2_{0}}\right)} \sum_{n_{3}=0}^{N_{3}-1} e^{j 2 \pi n_{3}\left(f_{3_{0}}-f_{3_{0_{l}}}\right)}
$$

It should be noted that $\mathbf{A}$ is a dense matrix having elements as the product and sum of complex numbers with a modulus of unity. Hence, the condition number of $\mathbf{A}$, which is the 
ratio of the largest to the smallest singular value in the singular value decomposition of the matrix, should be investigated with the variation of $L$ for stability of the algorithm.

First it is shown that $a_{k l}$ is bounded:

$$
\begin{aligned}
\left|a_{k l}\right| & =\left|\sum_{n_{1}=0}^{N_{1}-1} e^{j 2 \pi n_{1}\left(f_{1_{0_{k}}}-f_{1_{0_{l}}}\right)}\right|\left|\sum_{n_{2}=0}^{N_{2}-1} e^{j 2 \pi n_{2}\left(f_{2_{0_{k}}}-f_{2_{0_{l}}}\right)}\right||| \sum_{n_{3}=0}^{N_{3}-1} e^{j 2 \pi n_{3}\left(f_{3_{0_{k}}}-f_{3_{0_{l}}}\right)} \mid \\
& \leq \sum_{n_{1}=0}^{N_{1}-1}\left|e^{j 2 \pi n_{1}\left(f_{1_{0_{k}}}-f_{1_{0_{l}}}\right)}\right| \sum_{n_{2}=0}^{N_{2}-1}\left|e^{j 2 \pi n_{2}\left(f_{2_{0_{k}}}-f_{2_{0_{l}}}\right)}\right| \sum_{n_{3}=0}^{N_{3}-1}\left|e^{j 2 \pi n_{3}\left(f_{3_{0_{k}}}-f_{3_{0_{l}}}\right)}\right| \\
& \leq N_{1} N_{2} N_{3}
\end{aligned}
$$

The constant value of $a_{k l}=N_{1} N_{2} N_{3}$ appears on the diagonal of the symmetric matrix $\mathbf{A}$. All other entries of this matrix have a smaller magnitude than this constant as shown in (15). Although the proof of the non-singularity of $\mathbf{A}$ is not discussed here; however, we would like to report that we never encountered any unstable case during the simulations using different values of azimuth and elevation angles or different values of the number of antenna elements or the parameter $L$.

Now, we can use (9) and (10) to derive

$$
\mathbf{H}_{\mathbf{0}}=\mathbf{A b}
$$

Thus, $\mathbf{b}$ is obtained as follows:

$$
\mathbf{b}=\mathbf{A}^{-1} \mathbf{H}_{\mathbf{0}}
$$

In (17) we have assumed that $\mathbf{A}$ is not singular, so that its inverse exists. The proof of the nonsingularity of $\mathbf{A}$ is impossible or extremely difficult as the elements of this matrix are functions of the selection of the azimuth and elevation angles, the choice of $L$ and the dimensions of the array $N_{1}, N_{2}, N_{3}$. Simulation results with several values of these parameters never showed any difficulty in the calculations. The final step will be to calculate $C_{n_{1} n_{2} n_{3}}$ from the formula in (13).

Fig. 2 Space covered by $\left(f_{1}, f_{2}, f_{3}\right)$ and different octants

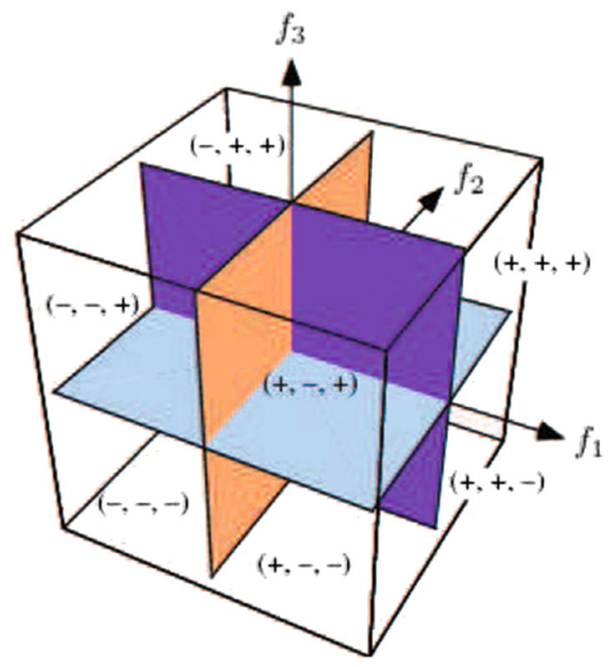


We now discuss the allocation of $L$ points $\left(f_{1_{0_{l}}}, f_{2_{0_{l}}}, f_{3_{0_{l}}}\right)$ with the corresponding values of $H\left(f_{1_{0_{l}}}, f_{2_{0_{l}}}, f_{3_{0_{l}}}\right)$ in the $\left(f_{1}, f_{2}, f_{3}\right)$ space. The simplest but non-trivial way to perform this allocation is $L=2$. The selection of these two points should be appropriate in order to generate a set of real coefficients $C_{n_{1} n_{2} n_{3}}$. For this purpose, as shown in Fig. 2, we need to select two points $P_{1}$ and $P_{2}$ symmetrically located in octant 1 and 7 , ladled by $(+,+,+)$ and $(-,-,-)$, respectively.

$$
\begin{aligned}
& P_{1}:\left(f_{1_{0_{1}}}, f_{2_{0_{1}}}, f_{3_{0_{1}}}\right)=\left(f_{1_{0}}, f_{2_{0}}, f_{3_{0}}\right) \\
& P_{2}: \quad\left(f_{1_{0_{2}}}, f_{2_{0_{2}}}, f_{3_{0_{2}}}\right)=\left(-f_{1_{0}},-f_{2_{0}},-f_{3_{0}}\right)
\end{aligned}
$$

Then, values at these points:

$$
\mathbf{H}_{\mathbf{0}}=[1,1]^{T}
$$

The symmetric selection guarantees that the coefficients are real and also that we have a unit gain at the desired point on the $\left(f_{1}, f_{2}, f_{3}\right)$ space. More complex choices can be made by adding several symmetric zeros to (18). For example, we can apply permutation rule to $\left( \pm f_{1}, \pm f_{2}, \pm f_{3}\right)$ and increase the number of critical points to $L=18$ as follows:

$$
\begin{array}{llll}
\left(f_{1_{0}}, f_{2_{0}}, f_{3_{0}}\right), \quad\left(-f_{1_{0}},-f_{2_{0}},-f_{3_{0}}\right), & \left(f_{2_{0}}, f_{3_{0}},-f_{1_{0}}\right), & \left(-f_{2_{0}},-f_{3_{0}}, f_{1_{0}}\right) \\
\left(f_{2_{0}},-f_{3_{0}}, f_{1_{0}}\right), \quad\left(-f_{2_{0}}, f_{3_{0}},-f_{1_{0}}\right), & \left(f_{2_{0}},-f_{3_{0}},-f_{1_{0}}\right), & \left(-f_{2_{0}}, f_{3_{0}}, f_{1_{0}}\right) \\
\left(f_{2_{0}}, f_{3_{0}}, f_{1_{0}}\right), \quad\left(-f_{2_{0}},-f_{3_{0}},-f_{1_{0}}\right), & \left(f_{3_{0}}, f_{1_{0}},-f_{2_{0}}\right), & \left(-f_{3_{0}},-f_{1_{0}},-f_{2_{0}}\right) \\
\left(-f_{3_{0}},-f_{1_{0}}, f_{2_{0}}\right), \quad\left(f_{3_{0}}, f_{1_{0}},-f_{2_{0}}\right), & \left(f_{3_{0}},-f_{1_{0}},-f_{2_{0}}\right), & \left(-f_{3_{0}}, f_{1_{0}}, f_{2_{0}}\right) \\
\left(f_{3_{0}}, f_{1_{0}}, f_{2_{0}}\right), \quad\left(-f_{3_{0}},-f_{1_{0}},-f_{2_{0}}\right) &
\end{array}
$$

For which we define:

$$
\mathbf{H}_{\mathbf{0}}=[1,1,0,0,0,0,0,0,0,0,0,0,0,0,0,0,0,0]^{T}
$$

That means the first two points $P_{1}$ and $P_{2}$ will be assigned an amplitude of one and the other 16 will have an amplitude of zero to improve the directivity of the resultant pattern.

The selection of $L$ is voluntary, however, to assure real coefficients for array elements, $L$ needs to be even. In order to complete the design with any other even values of $L$ between 2 and 18 (which is the maximum number we can generate by permutation of $\left( \pm f_{1}, \pm f_{2}, \pm f_{3}\right)$ ) we only need to keep the first $L$ values of (20) and discard the rest. Similarly, $\mathbf{H}_{\mathbf{0}}$ in (21) will only have two ones and $L-2$ zeros.

\section{Simulation results}

For the first simulation, we assume the simplest case of $L=2$ as in (18) and (19). The array configuration consists of $4 \times 4 \times 4$ elements. The desired azimuth and elevation angles are $\theta=70^{\circ}$ and $\phi=-50^{\circ}$. The lowest and highest operational frequencies have been considered as $3 \mathrm{GHz}$ and $4 \mathrm{GHz}$, respectively. This will generate a fractional bandwidth of about $30 \%$. We also assume that the inter-element spacing of the array is $d_{1}=d_{2}=d_{3}=3.75 \mathrm{~cm}$, which is half wavelength of the highest frequency.

To assign the values of $P_{1}$ and $P_{2}$, we have to calculate $f_{1_{0}}, f_{2_{0}}, f_{3_{0}}$ from (4). Then, $\mathbf{A}$ is constructed using (14) and $\mathbf{b}$ is calculated from (17). Finally, the coefficients $C_{n_{1} n_{2} n_{3}}$, for $1 \leq n_{1}, n_{2}, n_{3} \leq 4$, are computed from (13). Due to the symmetry of the selected points in the $\left(f_{1}, f_{2}, f_{3}\right)$ space, the values of $C_{n_{1} n_{2} n_{3}}$ are all real which reduces the computation load considerably and the array system will be fully spatially processed. 


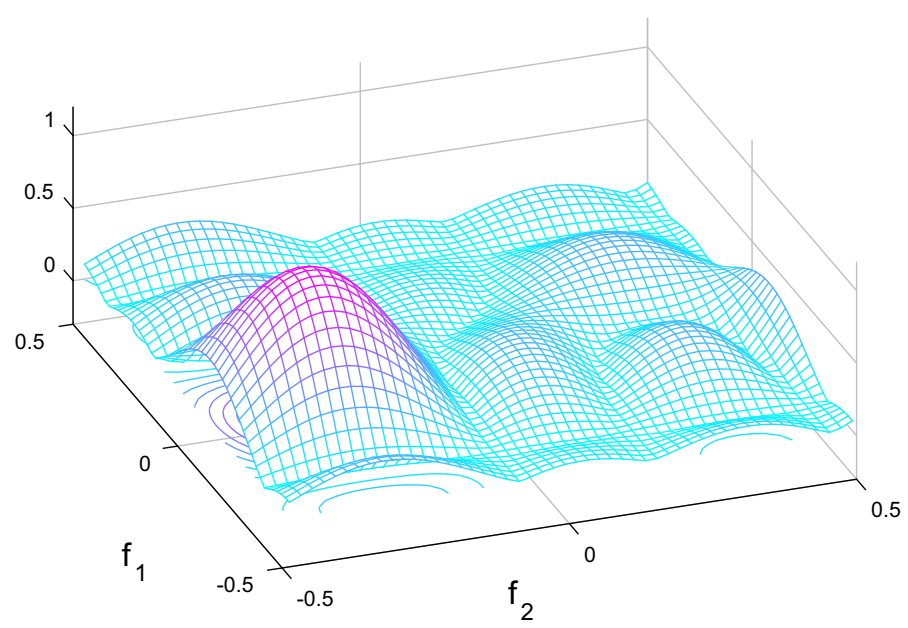

Fig. 3 Normalized plot of $H\left(f_{1}, f_{2}, f_{3}\right)$ as a function of $f_{1}$ and $f_{2}$. The global maximum is located at $\left(f_{1}=-0.1146, f_{2}=-0.3149\right)$

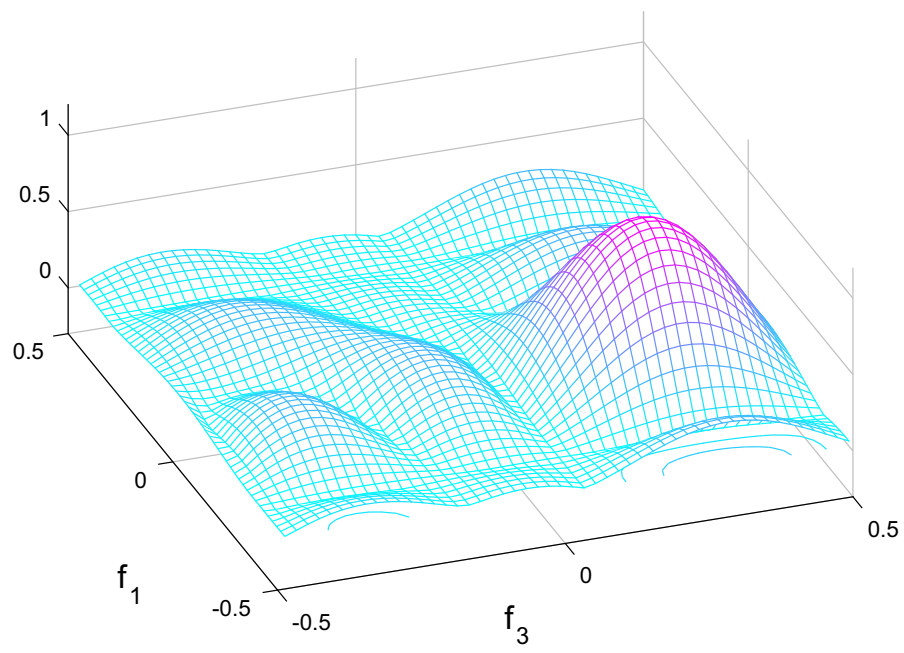

Fig. 4 Normalized plot of $H\left(f_{1}, f_{2}, f_{3}\right)$ as a function of $f_{1}$ and $f_{3}$. The global maximum is located at $\left(f_{1}=-0.1146, f_{3}=0.2812\right)$

Figure 3 shows a normalized plot of $H\left(f_{1}, f_{2}, f_{3}\right)$ calculated from (11) as a function of $f_{1}$ and $f_{2}$ while $f_{3}$ is kept constant to its nominal value when calculated at the midrange operational frequency. In the same way, Figs. 4 and 5 demonstrate the variation of $H\left(f_{1}, f_{2}, f_{3}\right)$ as $f_{2}$ and $f_{1}$, respectively, are maintained at their nominal values. All three figures show a relatively smooth variation of the magnitude close to the selected points based on (18). These mild variations are the key reasons for the frequency independence of the designed array, particularly near the desired direction.

Directional patterns of the three dimensional array with frequency variations from $3 \mathrm{GHz}$ to $4 \mathrm{GHz}$ are shown in Figs. 6 and 7 for azimuth and elevation angles, respectively. Both figures show the frequency independence of the design within the range and for the main 


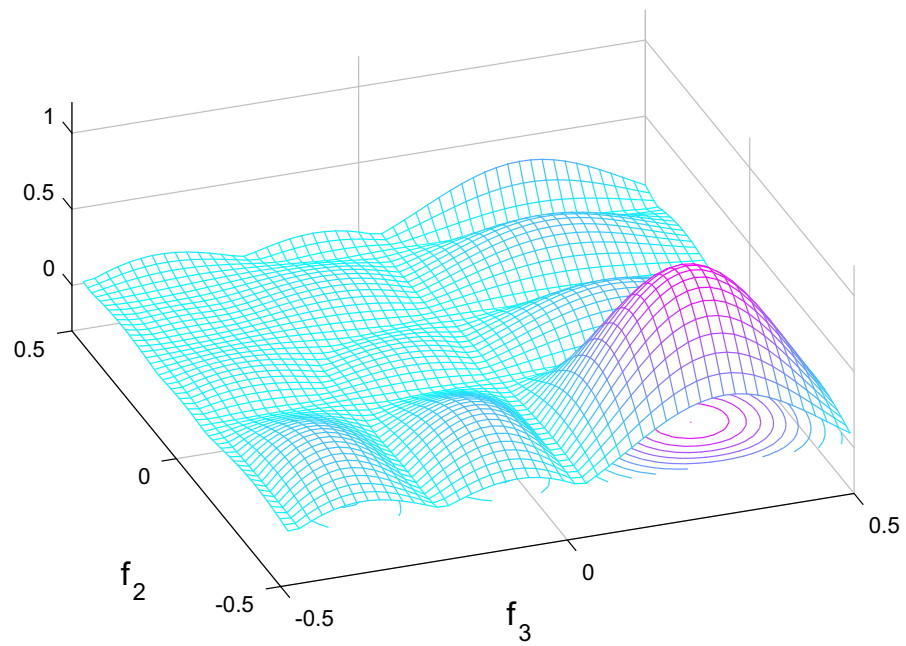

Fig. 5 Normalized plot of $H\left(f_{1}, f_{2}, f_{3}\right)$ as a function of $f_{2}$ and $f_{3}$. The global maximum is located at $\left(f_{2}=-0.3149, f_{3}=0.2812\right)$

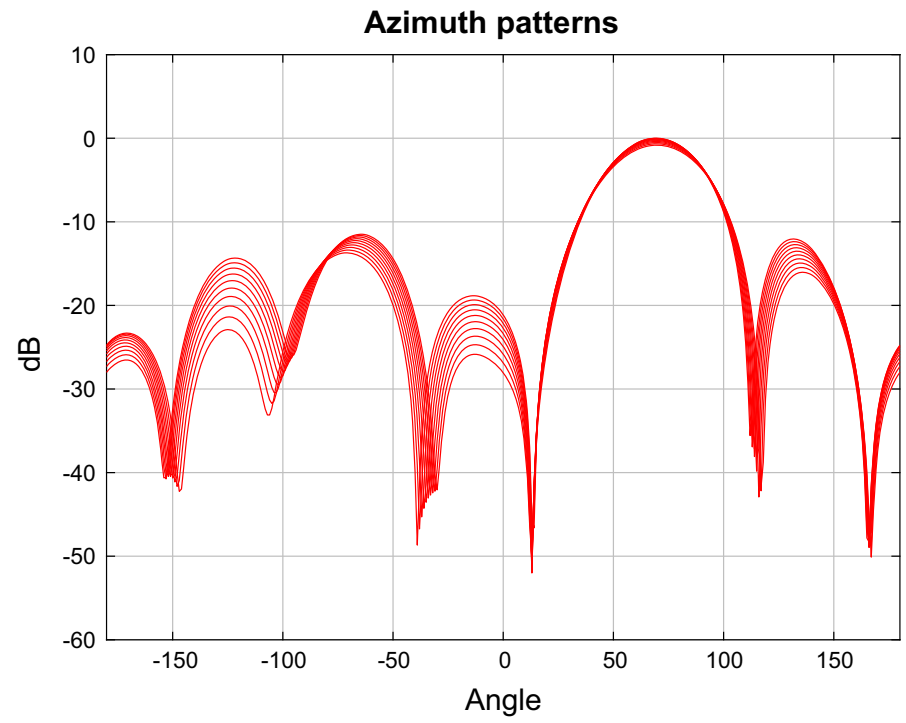

Fig. 6 Azimuth patterns for 3-4 GHz with the desired value of $\theta=70^{\circ}$ when $L=2$

lobe of the pattern. Relatively more variations are observed for the sidelobes but they all are happening below $10 \mathrm{~dB}$ attenuation.

Further simulations show that increasing $L=2$ to $L=18$ and using (20) together with (21) will decrease the beamwidth, sidelobe levels and frequency dependence by different factors that can be as high as $60 \%$ for sidelobes. These observations are presented in Figs. 8 and 9 . As we can notice, the $10 \mathrm{~dB}$ beamwidth has improved from $71^{\circ}$ to $59^{\circ}$ for the azimuth and from $52^{\circ}$ to $45^{\circ}$ for the elevation. This improvement is higher for the sidelobe attenuation levels which reduces from $-12 \mathrm{~dB}$ to $-20 \mathrm{~dB}$. 


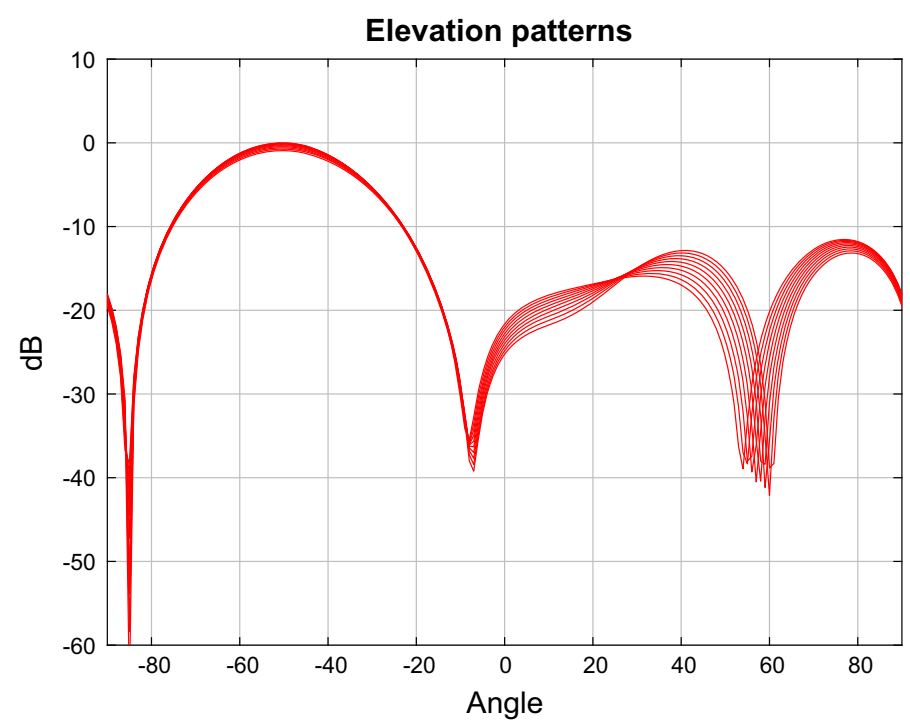

Fig. 7 Elevation patterns for 3-4 GHz with the desired value of $\phi=-50^{\circ}$ when $L=2$

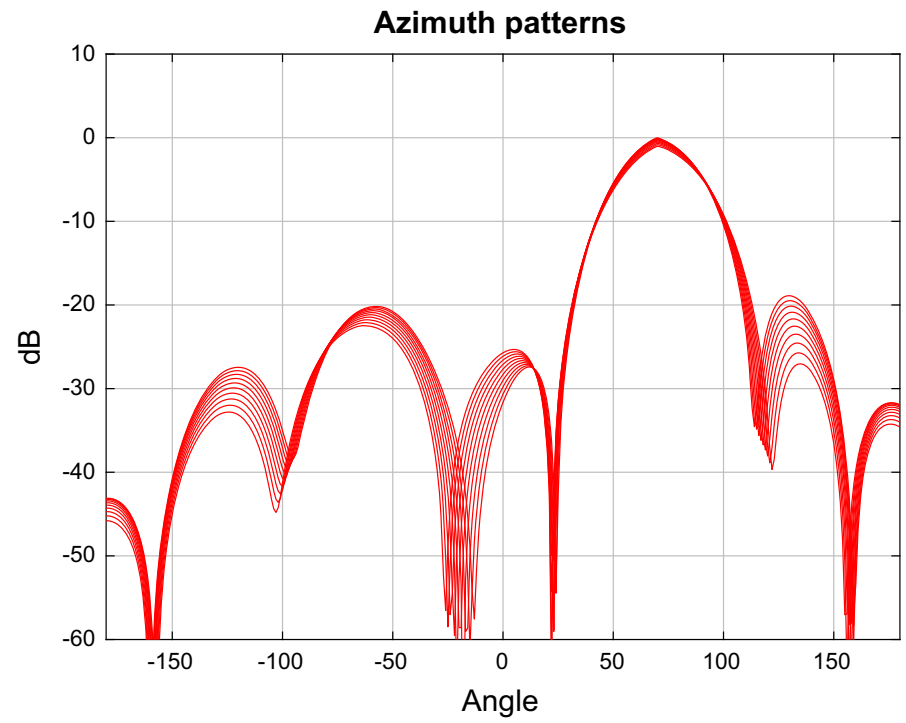

Fig. 8 Azimuth patterns for 3-4 GHz with the desired value of $\theta=70^{\circ}$ when $L=18$

For a comparison between the proposed algorithm and other methods employing cubic array structures, we have simulated the beamforming designs for the same number of elements and similar azimuth and elevation angles using the Fourier transform technique described in Liu (2010) and Liu and Weiss (2008) for calculation the weights connected to each element. The result of simulation is demonstrated in Figs. 10 and 11 for azimuth and elevation angles, respectively. These figures show that the sidelobe levels are only 6-8 dB lower than the main lobe, as compared to the 12-20 dB of the propose method in Figs. 6, 7, 8 and 9. 


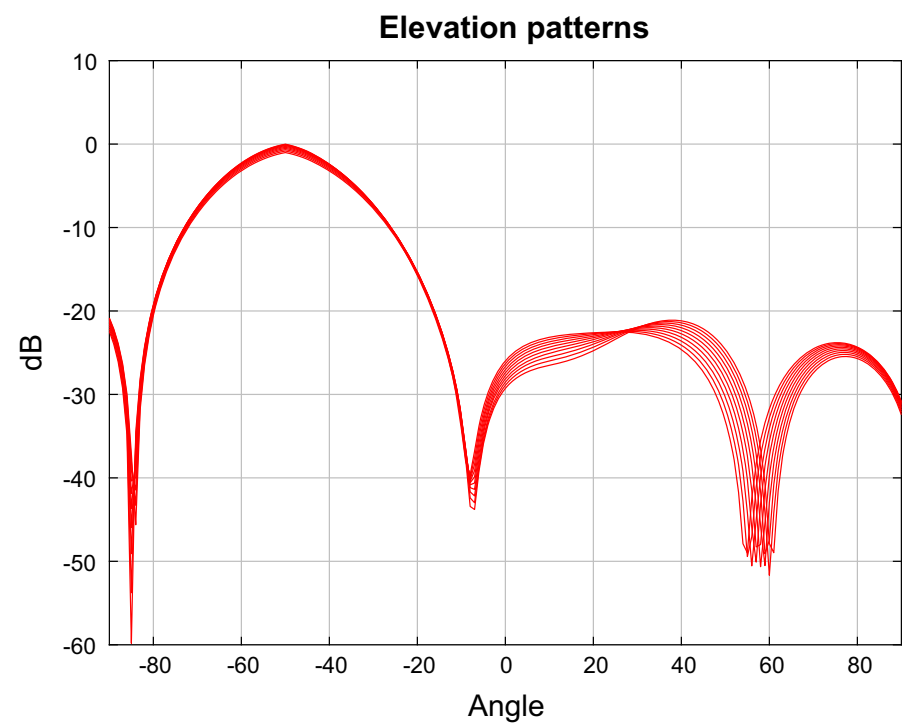

Fig. 9 Elevation patterns for $3-4 \mathrm{GHz}$ with the desired value of $\phi=-50^{\circ}$ when $L=18$

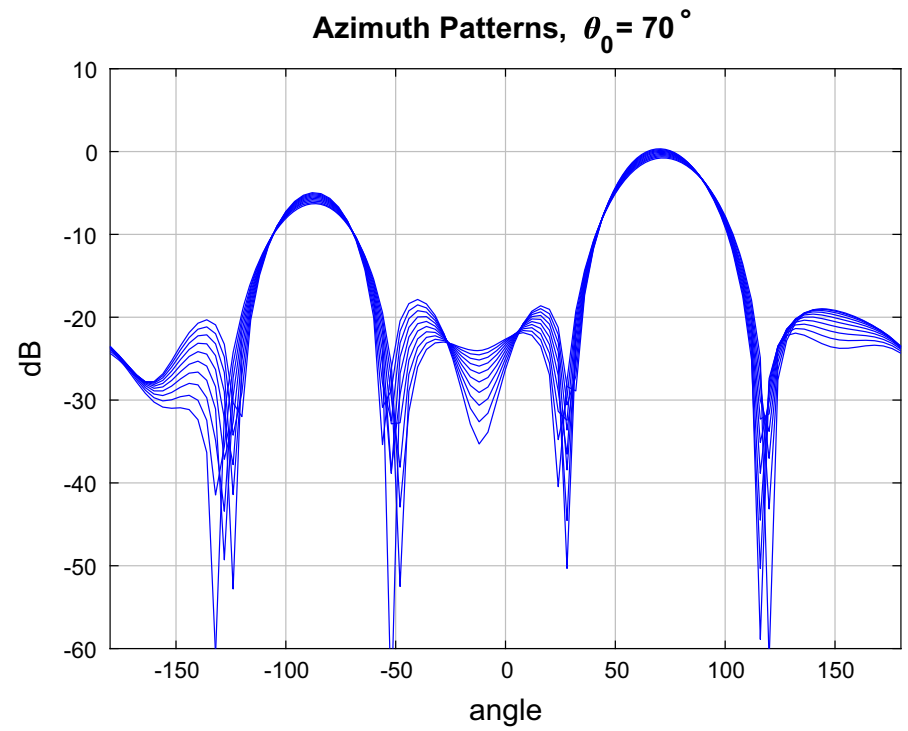

Fig. 10 Azimuth patterns for 3-4 GHz with the desired value of $\theta=70^{\circ}$ using Fourier transform algorithm

The second comparison is between the proposed method and the LMS adaptive algorithm for calculation of the real-valued coefficients. The LMS algorithm uses the following equation:

$$
C_{n_{1} n_{2} n_{3}}(t+\delta t)=C_{n_{1} n_{2} n_{3}}(t)+\mu e(t) s_{n_{1} n_{2} n_{3}}(t)
$$

to direct the mean squared error (MSE) toward zero, where $\mu$ is the step size or convergence factor and controls the convergence speed and stability of the calculations, $s_{n_{1} n_{2} n_{3}}(t)$ is the 


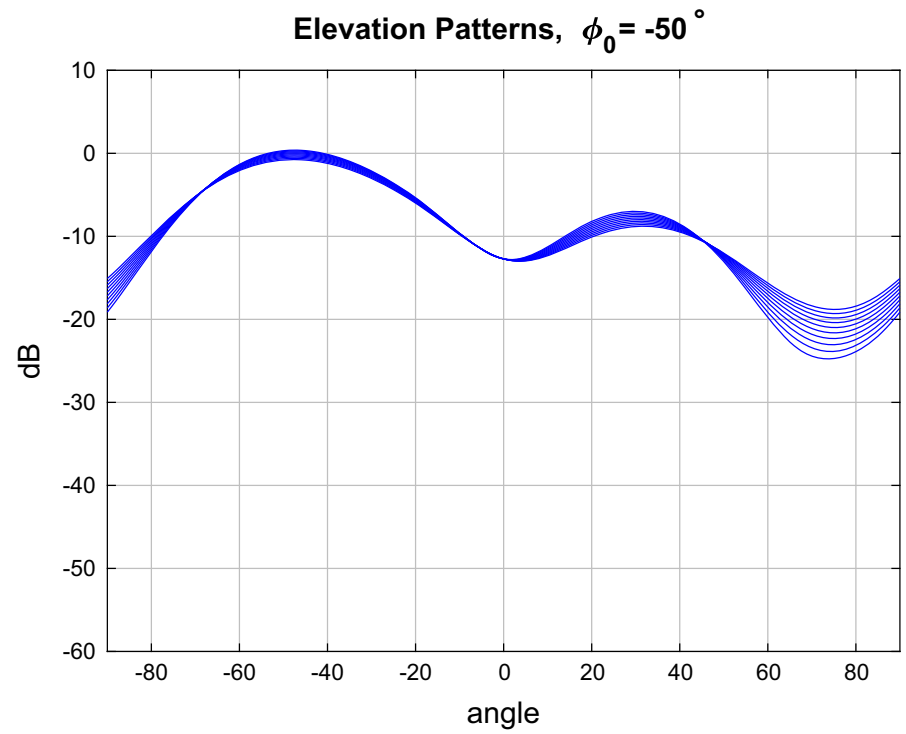

Fig. 11 Elevation patterns for 3-4 GHz with the desired value of $\phi=-50^{\circ}$ using Fourier transform algorithm

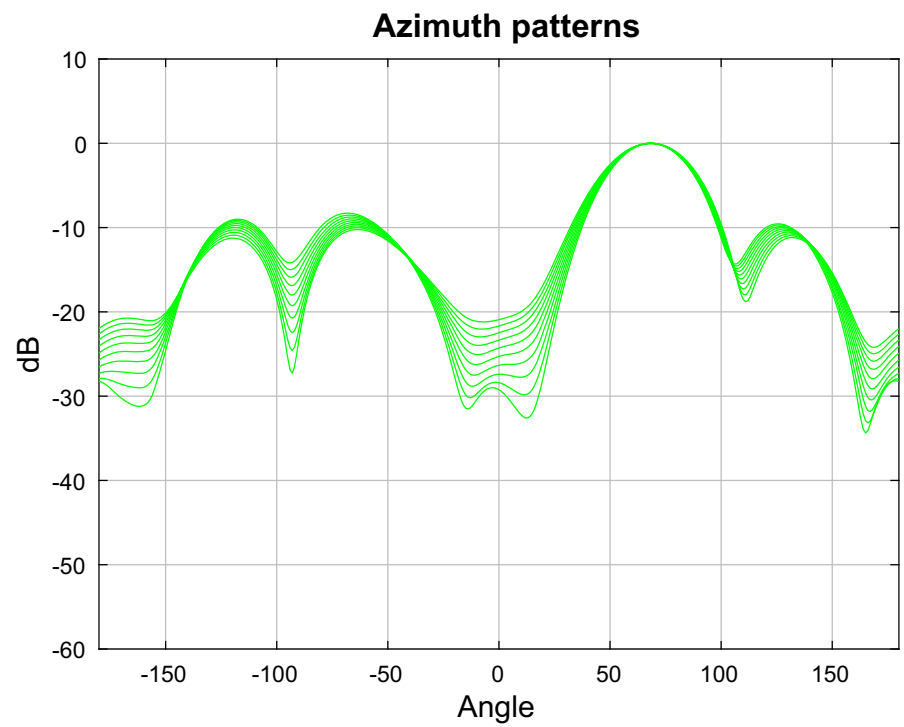

Fig. 12 Azimuth patterns for 3-4 GHz with the desired value of $\theta=70^{\circ}$ using LMS algorithm

input signal, $e(t)$ is the error and $\delta t$ is the time interval between two successive iterations. The result of simulation is demonstrated in Figs. 12 and 13 for azimuth and elevation angles, respectively. As it is observed, the results are not as good as the case of $L=2$ in Figs. 6 and 7 or the case of $L=18$ in Figs. 8 and 9 for sidelobe levels, $10 \mathrm{~dB}$ beamwidth and frequency independence. 


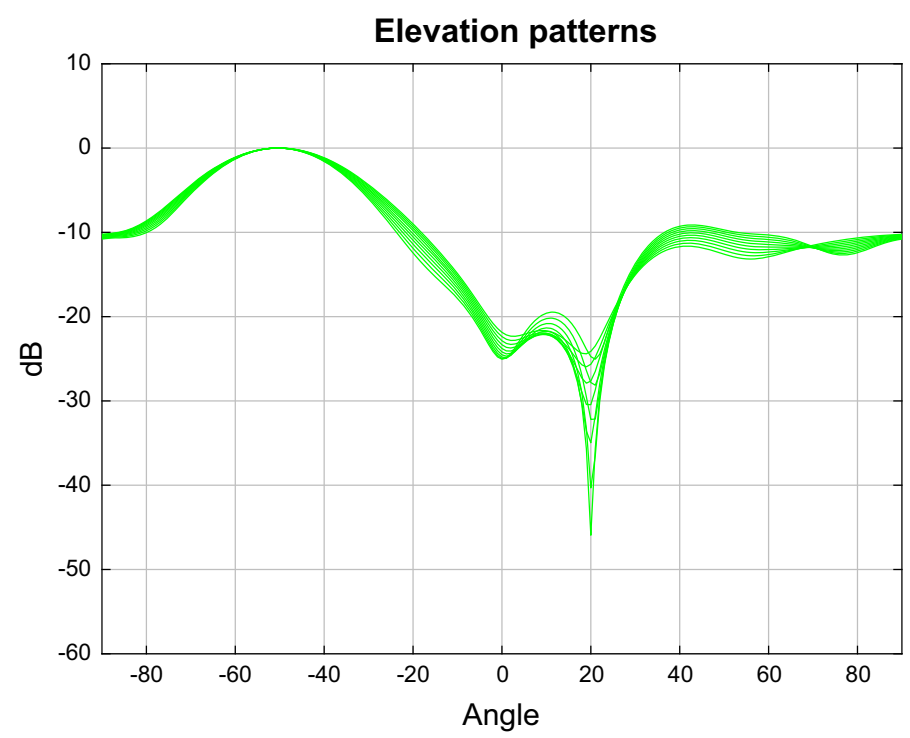

Fig. 13 Elevation patterns for $3-4 \mathrm{GHz}$ with the desired value of $\phi=-50^{\circ}$ using LMS algorithm

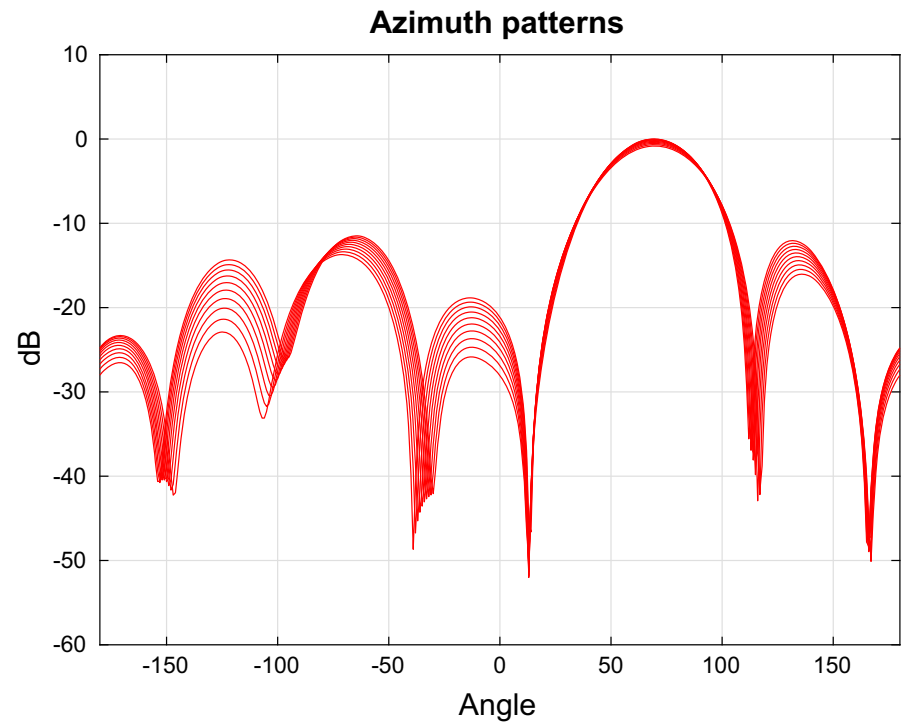

Fig. 14 Azimuth patterns for 7.5-10 GHz with the desired value of $\theta=70^{\circ}$ when $L=2$

Finally, to investigate the frequency range and bandwidth dependency of the proposed algorithm, Figs. 6 and 7 for azimuth and elevation angles of $\theta=70^{\circ}$ and $\phi=-50^{\circ}$, respectively, have been repeated for a higher and wider frequency range of $7.5-10 \mathrm{GHz}$ which is the higher portion of the ultra wideband (UWB) technology spectrum mask with a fractional bandwidth of about 30\%. The results are shown in Figs. 14 and 15, respectively. As we can see, as long as the fractional bandwidth is kept constant, the performance of the designed beamforming is basically the same across the whole UWB frequency domain. 


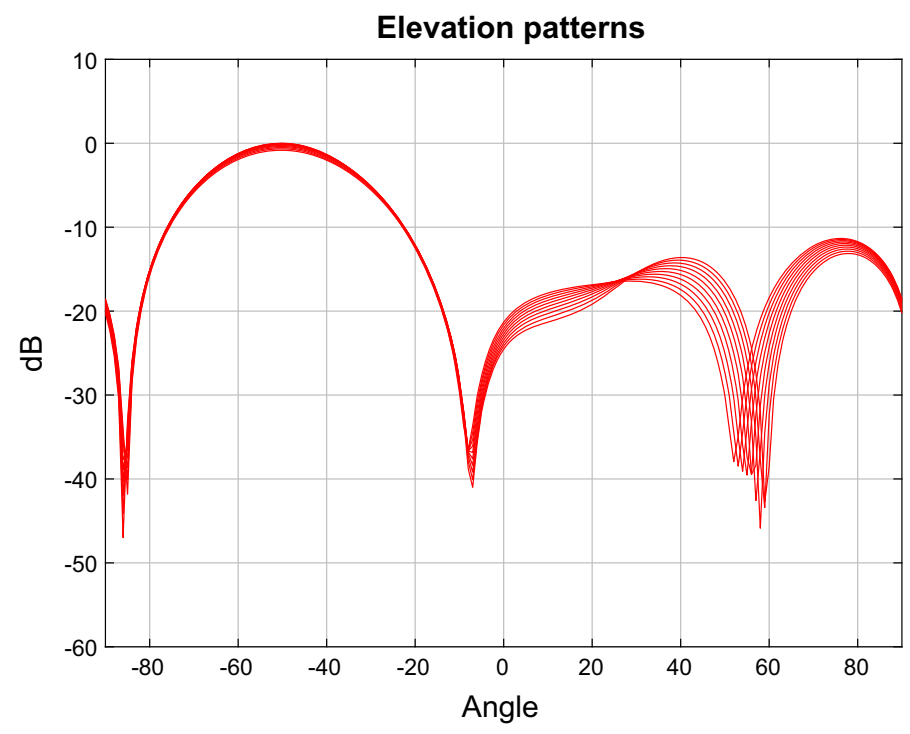

Fig. 15 Elevation patterns for 7.5-10 GHz with the desired value of $\phi=-50^{\circ}$ when $L=2$

\section{Conclusions}

A simple method for the design of a cubic array system with a real-valued multiplier for each antenna element was presented. The fully spatial beamformer can steer the beam toward both azimuth and elevation directions covering the whole three dimensional space. The configuration benefits from the frequency invariance of the patterns within a relatively large fractional bandwidth of about $30 \%$, which may be used in the whole UWB spectrum for different applications. The real multipliers were calculated based on matrix manipulation methods. However, the dimension of the matrices and vectors used in the computations can be much lower than the total number of antenna elements. Simulation results show that, even with the minimum number of required focal points $(L=2)$, the designed array shows an appropriate level of directivity and frequency independence for a fractional bandwidth of $30 \%$ within the UWB spectral mask from 3 to $10 \mathrm{GHz}$. Moreover, a comparison was made with the Fourier transform based and adaptive LMS algorithm methods in terms of the directivity and sidelobe levels to show the advantages of the proposed algorithm.

Open Access This article is distributed under the terms of the Creative Commons Attribution 4.0 International License (http://creativecommons.org/licenses/by/4.0/), which permits unrestricted use, distribution, and reproduction in any medium, provided you give appropriate credit to the original author(s) and the source, provide a link to the Creative Commons license, and indicate if changes were made.

\section{References}

Allen, B., \& Ghavami, M. (2005). Adaptive array systems: Fundamentals and applications. London: Wiley. Garza, J., Panduro, M. A., Reyna, A., Mendez, A., \& Covarrubias, D. H. (2016). Design of 3D cubic antenna arrays with low side lobe level during beam-scanning. In Proceedings of Asia-Pacific conference on antennas and propagation. 
Ghavami, M. (2002). Wideband smart antenna theory using rectangular array structures. IEEE Transaction on Signal Processing, 50(9), 2143-2150.

Hawes, M., \& Liu, W. (2014). Sparse array design for wideband beamforming with reduced complexity in tapped delay-lines. IEEE Transaction on Audio, Speech, and Language Processing, 22(8), 1236-1247.

Liang, L., \& Hum, S. V. (2011). Experimental characterization of UWB beamformers based on multidimensional beam filters. IEEE Transaction on Antenna and Propagation, 59(9), 304-309.

Liu, W. (2010). Wideband beamforming concepts and techniques. London: Wiley.

Liu, W., \& Weiss, S. (2008). Design of frequency invariant beamformers for broadband arrays. IEEE Transactions on Signal Processing, 56(2), 855-860.

Overturf, D., Buchanan, K., Jensen, J., Flores-Molina, C., Wheeland, S., \& Huff, G. H. (2017). Investigation of beamforming patterns from volumetrically distributed phased arrays. In Proceedings of IEEE military communications conference (MILCOM) (pp. 817-822).

Srikanth, S., Chaya, N. S., Gurugopinath, S., \& George, K. (2017). Optimal detection of a particle source using cubical and spherical directional detector arrays. In Proceedings of American control conference $(A C C)$.

Widrow, B., \& Stearns, S. D. (1985). Adaptive signal processing. Englewood Cliffs, NJ: Prentice-Hall.

Zhao, Y. Q., \& Peng, Z. (2009). Three-dimensional phased array antenna analysis and simulation. In Proceedings of antenna, propagation and EMC technologies for wireless communications: IEEE international symposium on microwave.

Publisher's Note Springer Nature remains neutral with regard to jurisdictional claims in published maps and institutional affiliations.

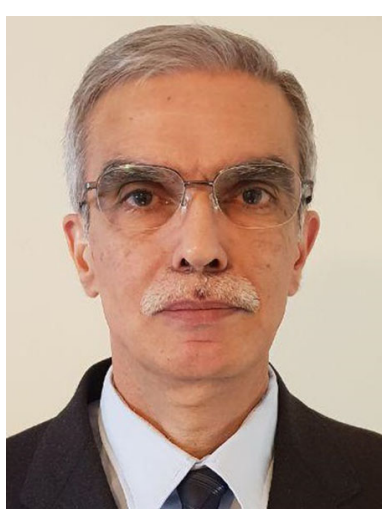

Mohammad Ghavami is a professor of Telecommunications at the London South Bank University. Prior to this appointment, he was with Kings College London from 2002 to 2010 and Sony Computer Science Laboratories in Tokyo from 2000 to 2002. He authored the books Ultra-Wideband Signals and Systems, and Adaptive Antenna Systems, and has published over 150 technical papers mainly related to UWB and its medical applications. He won the esteemed European Information Society Technologies prize in 2005 and two invention awards from Sony. He has been the Guest Editor of the IET Proceedings Communications, Special Issue on Ultra-Wideband Systems and the Associate Editor of the Special Issue of IEICE Journal on UWB Communications. He is a senior member of IEEE. 